\title{
Analysis System of Speech Acts and Discourse Structures Using Maximum Entropy Model*
}

\author{
Won Seug Choi, Jeong-Mi Cho and Jungyun Seo \\ Dept. of Computer Science, Sogang University \\ Sinsu-dong 1, Mapo-gu \\ Seoul, Korea, 121-742 \\ \{dolhana,jmcho\}@nlprep.sogang.ac.kr, seojy@ccs.sogang.ac.kr
}

\begin{abstract}
We propose a statistical dialogue analysis model to determine discourse structures as well as speech acts using maximum entropy model. The model can automatically acquire probabilistic discourse knowledge from a discourse tagged corpus to resolve ambiguities. We propose the idea of tagging discourse segment boundaries to represent the structural information of discourse. Using this representation we can effectively combine speech act analysis and discourse structure analysis in one framework.
\end{abstract}

\section{Introduction}

To understand a natural language dialogue, a computer system must be sensitive to the speaker's intentions indicated through utterances. Since identifying the speech acts of utterances is very important to identify speaker's intentions, it is an essential part of a dialogue analysis system. It is difficult, however, to infer the speech act from a surface utterance since an utterance may represent more than one speech act according to the context. Most works done in the past on the dialogue analysis has analyzed speech acts based on knowledge such as recipes for plan inference and domain specific knowledge (Litman (1987), Caberry (1989), Hinkelman (1990), Lambert (1991), Lambert (1993), Lee (1998)). Since these knowledge-based models depend on costly hand-crafted knowledge, these models are difficult to be scaled up and expanded to other domains.

\footnotetext{
* This work was supported by KOSEF under the contract 97-0102-0301-3.
}

Recently, machine learning models using a discourse tagged corpus are utilized to analyze speech acts in order to overcome such problems (Nagata (1994a), Nagata (1994b), Reithinger (1997), Lee (1997), Samuel (1998)). Machine learning offers promise as a means of associating features of utterances with particular speech acts, since computers can automatically analyze large quantities of data and consider many different feature interactions. These models are based on the features such as cue phrases, change of speaker, short utterances, utterance length, speech acts tag $n$-grams, and word $n$-grams, etc. Especially, in many cases, the speech act of an utterance influenced by the context of the utterance, i.e., previous utterances. So it is very important to reflect the information about the context to the model.

Discourse structures of dialogues are usually represented as hierarchical structures, which reflect embedding sub-dialogues (Grosz (1986)) and provide very useful context for speech act analysis. For example, utterance 7 in Figure 1 has several surface speech acts such as acknowledge, inform, and response. Such an ambiguity can be solved by analyzing the context. If we consider the $n$ utterances linearly adjacent to utterance 7 , i.e., utterances 6,5 , etc., as context, we will get acknowledge or inform with high probabilities as the speech act of utterance 7. However, as shown in Figure 1, utterance 7 is a response utterance to utterance 2 that is hierarchically recent to utterance 7 according to the discourse structure of the dialogue. If we know the discourse structure of the dialogue, we can determine the speech act of utterance 7 as response. 
Some researchers have used the structural information of discourse to the speech act analysis (Lee (1997), Lee (1998)). It is not, however, enough to cover various dialogues since they used a restricted rule-based model such as RDTN (Recursive Dialogue Transition Networks) for discourse structure analysis. Most of the previous related works, to our knowledge, tried to determine the speech act of an utterance, but did not mention about statistical models to determine the discourse structure of a dialogue.

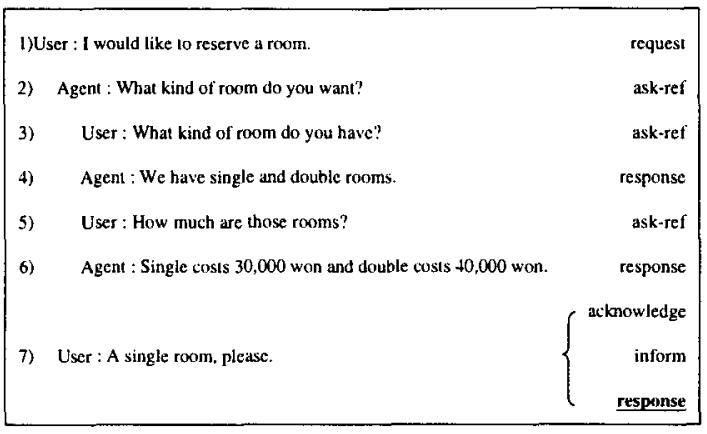

Figure 1: An example of a dialogue with speech acts

In this paper, we propose a dialogue analysis model to determine both the speech acts of utterances and the discourse structure of a dialogue using maximum entropy model. In the proposed model, the speech act analysis and the discourse structure analysis are combined in one framework so that they can easily provide feedback to each other. For the discourse structure analysis, we suggest a statistical model with discourse segment boundaries (DSBs) similar to the idea of gaps suggested for a statistical parsing (Collins (1996)). For training, we use a corpus tagged with various discourse knowledge. To overcome the problem of data sparseness, which is common for corpus-based works, we use split partial context as well as whole context.

After explaining the tagged dialogue corpus we used in section 1, we discuss the statistical models in detail in section 2 . In section 3 , we explain experimental results. Finally, we conclude in section 4 .

\section{Discourse tagging}

In this paper, we use Korean dialogue corpus transcribed from recordings in real fields such as hotel reservation, airline reservation and tour reservation. This corpus consists of 528 dialogues, 10,285 utterances (19.48 utterances per dialogue). Each utterance in dialogues is manually annotated with discourse knowledge such as speaker (SP), syntactic pattern (ST), speech acts (SA) and discourse structure (DS) information. Figure 2 shows a part of the annotated dialogue corpus ${ }^{1}$. SP has a value either "User" or "Agent" depending on the speaker.

\begin{tabular}{|c|c|}
\hline 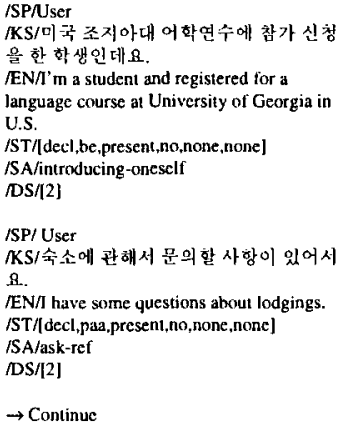 & 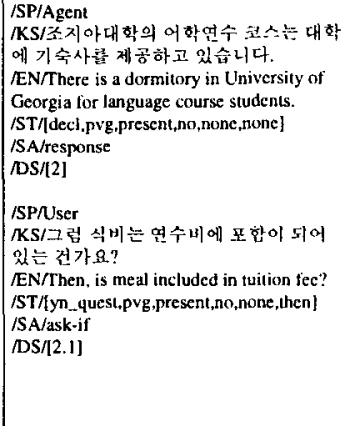 \\
\hline
\end{tabular}

Figure 2: A part of the annotated dialogue corpus

The syntactic pattern consists of the selected syntactic features of an utterance, which approximate the utterance. In a real dialogue, a speaker can express identical contents with different surface utterances according to a personal linguistic sense. The syntactic pattern generalizes these surface utterances using syntactic features. The syntactic pattern used in (Lee (1997)) consists of four syntactic features such as Sentence Type, Main-Verb, Aux-Verb and Clue-Word because these features provide strong cues to infer speech acts. We add two more syntactic features, Tense and Negative Sentence, to the syntactic pattern and elaborate the values of the syntactic features. Table 1 shows the syntactic features of a syntactic pattern with possible values. The syntactic features are automatically extracted from the corpus using a conventional parser (Kim (1994)).

Manual tagging of speech acts and discourse structure information was done by graduate students majoring in dialogue analysis and postprocessed for consistency. The classification of speech acts is very subjective without an agreed criterion. In this paper, we classified the 17 types of speech acts that appear in the dialogue

1 KS represents the Korean sentence and EN represents the translated English sentence. 
corpus. Table 2 shows the distribution of speech acts in the tagged dialogue corpus.

Discourse structures are determined by focusing on the subject of current dialogue and are hierarchically constructed according to the subject. Discourse structure information tagged in the corpus is an index that represents the hierarchical structure of discourse reflecting the depth of the indentation of discourse segments. The proposed system transforms this index information to discourse segment boundary (DSB) information to acquire various statistical information. In section 2.2.1, we will describe the DSBs in detail.

\begin{tabular}{|c|l|l|}
\hline Syntactic fearure & \multicolumn{1}{|c|}{ Values } & \multicolumn{1}{|c|}{ Notes } \\
\hline Sentence Type & $\begin{array}{l}\text { decl, imperative, } \\
\text { wh_question, yn_question }\end{array}$ & The mood of an utterance \\
\hline Main-Verb & $\begin{array}{l}\text { pvg, pvd, paa, pad, be, } \\
\text { know, ask, ctc. } \\
\text { (total 88 kinds) }\end{array}$ & $\begin{array}{l}\text { The type of the main verb. For } \\
\text { special verbs, lexical items are } \\
\text { used. }\end{array}$ \\
\hline Tense & past, present, future. & The tense of an uterance \\
\hline Negative Sentence & Yes or No & Yes if an utterance is negative. \\
\hline Aux-Verb & $\begin{array}{l}\text { serve, seem, want, will, } \\
\text { etc. (total 31 kinds) }\end{array}$ & The modality of an utterance. \\
\hline Clue-Word & $\begin{array}{l}\text { Yes, No, OK., etc. } \\
\text { (total 26 kinds) }\end{array}$ & $\begin{array}{l}\text { The special word used in the } \\
\text { utterance having panticular } \\
\text { speech acts. }\end{array}$ \\
\hline
\end{tabular}

Table I: Syntactic features used in the syntactic pattern

\begin{tabular}{|l|c|l|c|}
\hline Speech Act Type & Ratio(\%) & Speech Act Type & Ratio(\%) \\
\hline Accept & 2.49 & Introducing-oneself & 6.75 \\
\hline Acknowledge & 5.75 & Offer & 0.40 \\
\hline Ask-confirm & 3.16 & Opening & 6.58 \\
\hline Ask-if & 5.36 & Promise & 2.42 \\
\hline Ask-ref & 13.39 & Reject & 1.07 \\
\hline Closing & 3.39 & Request & 4.96 \\
\hline Correct & 0.03 & Response & 24.73 \\
\hline Expressive & 5.64 & Suggest & 1.98 \\
\hline Inform & 11.90 & Total & 100.00 \\
\hline
\end{tabular}

Table 2: The distribution of speech acts in corpus

\section{$2 \quad$ Statistical models}

We construct two statistical models: one for speech act analysis and the other for discourse structure analysis. We integrate the two models using maximum entropy model. In the following subsections, we describe these models in detail.

\subsection{Speech act analysis model}

Let $U_{1, n}$ denote a dialogue which consists of a sequence of $n$ utterances, $U_{1}, U_{2}, \ldots, U_{n}$, and let
$S_{i}$ denote the speech act of $U_{i}$. With these notations, $P\left(S_{i} \mid U_{1, i}\right)$ means the probability that $S_{i}$ becomes the speech act of utterance $U_{i}$ given a sequence of utterances $U_{1}, U_{2}, \ldots, U_{i}$. We can approximate the probability $P\left(S_{i} \mid U_{1, i}\right)$ by the product of the sentential probability $P\left(U_{i} \mid S_{i}\right)$ and the contextual probability $P\left(S_{i} \mid U_{1, i-1,} S_{1, i-1}\right)$. Also we can approximate $\quad P\left(S_{i} \mid U_{1, i-1}, S_{1, i-1}\right) \quad$ by $P\left(S_{i} \mid S_{1, i-1}\right)$ (Charniak (1993)).

$P\left(S_{i} \mid U_{1, i}\right) \approx P\left(S_{i} \mid S_{1, i-1}\right) P\left(U_{i} \mid S_{i}\right)$

It has been widely believed that there is a strong relation between the speaker's speech act and the surface utterances expressing that speech act (Hinkelman (1989), Andernach (1996)). That is, the speaker utters a sentence, which most well expresses his/her intention (speech act) so that the hearer can easily infer what the speaker's speech act is. The sentential probability $P\left(U_{i} \mid S_{i}\right)$ represents the relationship between the speech acts and the features of surface sentences. Therefore, we approximate the sentential probability using the syntactic pattern $P_{i}$.

$$
P\left(U_{i} \mid S_{i}\right) \approx P\left(P_{i} \mid S_{i}\right)
$$

The contextual probability $P\left(S_{i} \mid S_{1, i-1}\right)$ is the probability that utterance with speech act $S_{i}$ is uttered given that utterances with speech act $S_{1}, S_{2}, \ldots, S_{i-1}$ were previously uttered. Since it is impossible to consider all preceding utterances $S_{1}, S_{2}, \ldots, S_{i-1}$ as contextual information, we use the $n$-gram model. Generally, dialogues have a hierarchical discourse structure. So we approximate the context as speech acts of $n$ utterances that are hierarchically recent to the utterance. An utterance $\mathbf{A}$ is hierarchically recent to an utterance $\mathbf{B}$ if $\mathbf{A}$ is adjacent to $\mathbf{B}$ in the tree structure of the discourse (Walker (1996)). Equation (3) represents the approximated contextual probability in the case of using trigram where $U_{j}$ and $U_{k}$ are hierarchically recent to the utterance $U_{i}$, where $1 \leq j \leq k \leq i-1$. 


$$
P\left(S_{i} \mid S_{1, i-1}\right) \approx P\left(S_{i} \mid S_{j}, S_{k}\right)
$$

As a result, the statistical model for speech act analysis is represented in equation (4).

$$
\begin{aligned}
P\left(S_{i} \mid U_{1, i}\right) & \approx P\left(S_{i} \mid S_{1, i-1}\right) P\left(U_{i} \mid S_{i}\right) \\
& \approx P\left(S_{i} \mid S_{j}, S_{k}\right) P\left(P_{i} \mid S_{i}\right)
\end{aligned}
$$

\subsection{Discourse structure analysis model}

\subsubsection{Discourse segment boundary tagging}

We define a set of discourse segment boundaries (DSBs) as the markers for discourse structure tagging. A DSB represents the relationship between two consecutive utterances in a dialogue. Table 3 shows DSBs and their meanings, and Figure 3 shows an example of DSB tagged dialogue.

\begin{tabular}{|l|l|}
\hline DSB & Meaning \\
\hline $\mathrm{DE}$ & Start a new dialogue \\
\hline $\mathrm{DC}$ & Continue a dialogue \\
\hline $\mathrm{SS}$ & Start a sub-dialogue \\
\hline$n \mathrm{E}$ & End $n$ level sub-dialogues \\
\hline$n \mathrm{~B}$ & $n \mathrm{E}$ and then $\mathrm{SS}$ \\
\hline
\end{tabular}

Table 3: DSBs and their meanings

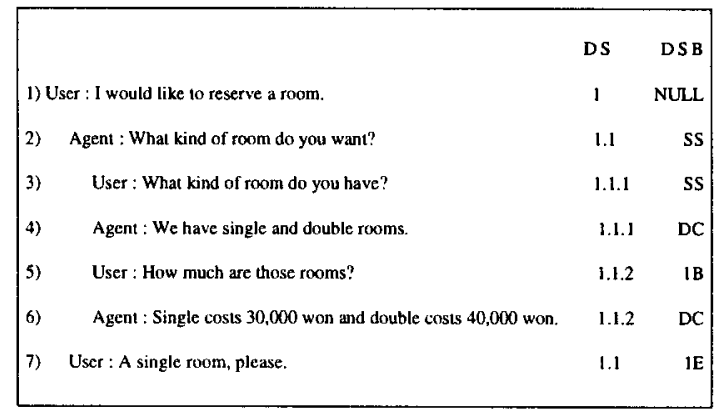

Figure 3: An example of DSB tagging

Since the DSB of an utterance represents a relationship between the utterance and the previous utterance, the DSB of utterance 1 in the example dialogue becomes NULL. By comparing utterance 2 with utterance 1 in Figure
3 , we know that a new sub-dialogue starts at utterance 2 . Therefore the DSB of utterance 2 becomes SS. Similarly, the DSB of utterance 3 is SS. Since utterance 4 is a response for utterance 3 , utterance 3 and 4 belong to the same discourse segment. So the DSB of utterance 4 becomes DC. Since a sub-dialogue of one level (i.e., the DS 1.1.2) consisting of utterances 3 and 4 ends, and new sub-dialogue starts at utterance 5 . Therefore, the DSB of utterance 5 becomes 1B. Finally, utterance 7 is a response for utterance 2 , i.e., the sub-dialogue consisting of utterances 5 and 6 ends and the segment 1.1 is resumed. Therefore the DSB of utterance 7 becomes $1 \mathrm{E}$.

\subsubsection{Statistical model for discourse structure analysis}

We construct a statistical model for discourse structure analysis using DSBs. In the training phase, the model transforms discourse structure (DS) information in the corpus into DSBs by comparing the DS information of an utterance with that of the previous utterance. After transformation, we estimate probabilities for DSBs. In the analyzing process, the goal of the system is simply determining the DSB of a current utterance using the probabilities. Now we describe the model in detail.

Let $G_{i}$ denote the DSB of $U_{i}$. With this notation, $P\left(G_{i} \mid U_{1, i}\right)$ means the probability that $G_{i}$ becomes the DSB of utterance $U_{i}$ given a sequence of utterances $U_{1}, U_{2}, \ldots, U_{i}$. As shown in the equation (5), we can approximate $P\left(G_{i} \mid U_{1, i}\right)$ by the product of the sentential probability $P\left(U_{i} \mid G_{i}\right)$ and the contextual probability $P\left(G_{i} \mid U_{1, i-1,} G_{1, i-1}\right)$ :

$$
\begin{aligned}
& P\left(G_{i} \mid U_{1, i}\right) \\
& \quad \approx P\left(G_{i} \mid U_{1, i-1}, G_{i, i-1}\right) P\left(U_{i} \mid G_{i}\right)
\end{aligned}
$$

In order to analyze discourse structure, we consider the speech act of each corresponding utterance. Thus we can approximate each utterance by the corresponding speech act in the sentential probability $P\left(U_{i} \mid G_{i}\right)$ :

$$
P\left(U_{i} \mid G_{i}\right) \approx P\left(S_{i} \mid G_{i}\right)
$$


Let $F_{i}$ be a pair of the speech act and DSB of $U_{1}$ to simplify notations:

$$
F_{i} \equiv\left(S_{i}, G_{i}\right)
$$

We can approximate the contextual probability $P\left(G_{i} \mid U_{1, i-1}, G_{1, i-1}\right)$ as equation (8) in the case of using trigram.

$$
\begin{aligned}
& P\left(G_{i} \mid U_{1, i-1}, G_{1, i-1}\right) \\
& \quad \approx P\left(G_{i} \mid F_{1, i-1}\right) \approx P\left(G_{i} \mid F_{i-2}, F_{i-1}\right)
\end{aligned}
$$

As a result, the statistical model for the discourse structure analysis is represented as equation (9).

$$
\begin{aligned}
& P\left(G_{i} \mid U_{1, i}\right) \\
& \quad \approx P\left(G_{i} \mid U_{1, i-1}, G_{1, i-1}\right) P\left(U_{i} \mid G_{i}\right) \\
& \approx P\left(G_{i} \mid F_{i-2,}, F_{i-1}\right) P\left(S_{i} \mid G_{i}\right)
\end{aligned}
$$

\subsection{Integrated dialogue analysis model}

Given a dialogue $U_{1, n}, P\left(S_{i}, G_{i} \mid U_{1, i}\right)$ means the probability that $S_{i}$ and $G_{i}$ will be, respectively, the speech act and the DSB of an utterance $U_{i}$ given a sequence of utterances $U_{1}, U_{2}, \ldots, U_{i}$. By using a chain rule, we can rewrite the probability as in equation (10).

$$
\begin{aligned}
& P\left(S_{i}, G_{i} \mid U_{1, i}\right) \\
& \quad=P\left(S_{i} \mid U_{1, i}\right) P\left(G_{i} \mid S_{i}, U_{1, i}\right)
\end{aligned}
$$

In the right hand side (RHS) of equation (10), the first term is equal to the speech act analysis model shown in section 2.1. The second term can be approximated as the discourse structure analysis model shown in section 2.2 because the discourse structure analysis model is formulated by considering utterances and speech acts together. Finally the integrated dialogue analysis model can be formulated as the product of the speech act analysis model and the discourse structure analysis model:

$$
\begin{aligned}
P( & \left.S_{i}, G_{i} \mid U_{1, i}\right) \\
\approx & P\left(S_{i} \mid U_{1, i}\right) P\left(G_{i} \mid U_{1, i}\right) \\
= & P\left(S_{i} \mid S_{j}, S_{k}\right) P\left(P_{i} \mid S_{i}\right) \\
& \times P\left(G_{i} \mid F_{i-2}, F_{i-1}\right) P\left(S_{i} \mid G_{i}\right)
\end{aligned}
$$

\subsection{Maximum entropy model}

All terms in RHS of equation (11) are represented by conditional probabilities. We estimate the probability of each term using the following representative equation:

$P(a \mid b)=\frac{P(a, b)}{\sum_{a^{\prime}} P\left(a^{\prime}, b\right)}$

We can evaluate $P(a, b)$ using maximum entropy model shown in equation (13) (Reynar 1997).

$$
\begin{aligned}
& P(a, b)=\pi \prod_{i=1}^{k} \alpha_{i}^{f_{i}(a, b)} \\
& \quad \text { where } 0<\alpha_{\mathrm{i}}<\infty, i=\{1,2, \ldots, k\}
\end{aligned}
$$

In equation (13), $a$ is either a speech act or a DSB depending on the term, $b$ is the context (or history) of $a, \pi$ is a normalization constant, and $\alpha_{i}$ is the model parameter corresponding to each feature function $f_{i \text {. }}$

In this paper, we use two feature functions: unified feature function and separated feature function. The former uses the whole context $b$ as shown in equation (12), and the latter uses partial context split-up from the whole context to cope with data sparseness problems. Equation (14) and (15) show examples of these feature functions for estimating the sentential probability of the speech act analysis model.

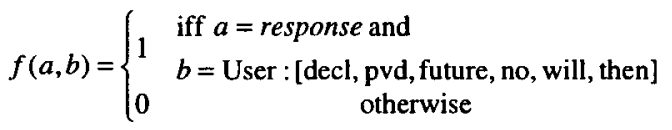

$$
\begin{aligned}
& f(a, b)= \begin{cases}1 & \text { iff } a=\text { response } \text { and } \\
0 & \text { SentenceType }(b)=\text { User }: \text { decl } \\
0 & \text { otherwise }\end{cases}
\end{aligned}
$$

Equation (14) represents a unified feature function constructed with a syntactic pattern 
having all syntactic features, and equation (15) represents a separated feature function constructed with only one feature, named Sentence Type, among all syntactic features in the pattern. The interpretation of the unified feature function shown in equation (14) is that if the current utterance is uttered by "User", the syntactic pattern of the utterance is [decl,pvd,future,no,will,then] and the speech act of the current utterance is response then $f(a, b)=1$ else $f(a, b)=0$. We can construct five more separated feature functions using the other syntactic features. The feature functions for the contextual probability can be constructed in similar ways as the sentential probability. Those are unified feature functions with feature trigrams and separated feature functions with distance-1 bigrams and distance-2 bigrams. Equation (16) shows an example of an unified feature function, and equation (17) and (18) which are delivered by separating the condition of $b$ in equation (16) show examples of separated feature functions for the contextual probability of the speech act analysis model.

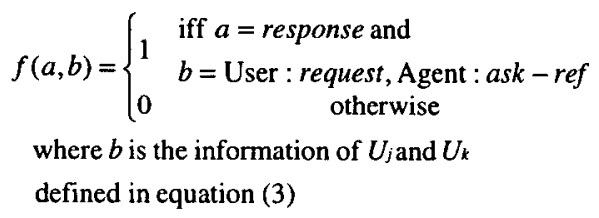

$f(a, b)=\left\{\begin{array}{cc}1 & \text { iff } a=\text { response } \text { and } \\ & b_{-1}=\text { Agent }: a s k-r e f \\ 0 & \text { otherwise }\end{array}\right.$

where $b_{-1}$ is the information of $U_{k}$ defined in equation (3)

$f(a, b)=\left\{\begin{array}{cc}1 & \text { iff } a=\text { response } \text { and } b_{-2}=\text { User : request } \\ 0 & \text { otherwise }\end{array}\right.$

where $b_{-2}$ is the information of $U_{j}$ defined in equation (3)

Similarly, we can construct feature functions for the discourse structure analysis model. For the sentential probability of the discourse structure analysis model, the unified feature function is identical to the separated feature function since the whole context includes only a speech act. Using the separated feature functions, we can solve the data sparseness problem when there are not enough training examples to which the unified feature function is applicable.

\section{Experiments and results}

In order to experiment the proposed model, we used the tagged corpus shown in section 1 . The corpus is divided into the training corpus with 428 dialogues, 8,349 utterances (19.51 utterances per dialogue), and the testing corpus with 100 dialogues, 1,936 utterances (19.36 utterances per dialogue). Using the Maximum Entropy Modeling Toolkit (Ristad 1996), we estimated the model parameter $\alpha_{i}$ corresponding to each feature function $f_{i}$ in equation (13).

We made experiments with two models for each analysis model. Model-I uses only the unified feature function, and Model-II uses the unified feature function and the separated feature function together. Among the ways to combine the unified feature function with the separated feature function, we choose the combination in which the separated feature function is used only when there is no training example applicable for the unified feature function.

First, we tested the speech act analysis model and the discourse analysis model. Table 4 and 5 show the results for each analysis model. The results shown in table 4 are obtained by using the correct structural information of discourse, i.e., DSB, as marked in the tagged corpus. Similarly those in table 5 are obtained by using the correct speech act information from the tagged corpus.

\begin{tabular}{|l|c|c|c|c|}
\hline & \multicolumn{2}{|c|}{ Accuracy (Closed test) } & \multicolumn{2}{c|}{ Accuracy (Open test) } \\
\hline Candidates & Top-1 & Top-3 & Top-1 & Top-3 \\
\hline Lee (1997) & $78.59 \%$ & $97.88 \%$ & - & - \\
\hline Samuel (1998) & & & $73.17 \%$ & \\
\hline Reithinger (1997) & & & $74.70 \%$ & \\
\hline Model I & $90.65 \%$ & $99.66 \%$ & $81.61 \%$ & $93.18 \%$ \\
\hline Model II & $90.65 \%$ & $99.66 \%$ & $\mathbf{8 3 . 3 7 \%}$ & $95.35 \%$ \\
\hline
\end{tabular}

Table 4. Results of speech act analysis

\begin{tabular}{|l|c|c|}
\hline & \multicolumn{2}{|c|}{ Accuracy(Open test) } \\
\hline Candidates & Top-1 & Top-3 \\
\hline Model I & $81.51 \%$ & $98.55 \%$ \\
\hline Model I & $83.21 \%$ & $99.02 \%$ \\
\hline
\end{tabular}

Table 5. Results of discourse structure analysis

In the closed test in table 4, the results of ModelI and Model-II are the same since the probabilities of the unified feature functions always exist in this case. As shown in table 4, the proposed models show better results than previous work, Lee (1997). As shown in table 4 and 5, Model-II shows better results than Model- 
I in all cases. We believe that the separated feature functions are effective for the data sparseness problem. In the open test in table 4 , it is difficult to compare the proposed model directly with the previous works like Samuel (1998) and Reithinger (1997) because test data used in those works consists of English dialogues while we use Korean dialogues. Furthermore the speech acts used in the experiments are different. We will test our model using the same data with the same speech acts as used in those works in the future work. We tested the integrated dialogue analysis model in which speech act and discourse structure analysis models are integrated. The integrated model uses Model-II for each analysis model because it showed better performance. In this model, after the system determing the speech act and DSB of an utterance, it uses the results to process the next utterance, recursively. The experimental results are shown in table 6.

As shown in table 6, the results of the integrated model are worse than the results of each analysis model. For top- 1 candidate, the performance of the speech act analysis fell off about $2.89 \%$ and that of the discourse structure analysis about $7.07 \%$. Nevertheless, the integrated model still shows better performance than previous work in the speech act analysis.

\begin{tabular}{|l|l|l|}
\hline & \multicolumn{2}{|l|}{ Accuracy(Open test) } \\
\hline Candidates & Top-1 & Top-3 \\
\hline $\begin{array}{l}\text { Result of speech act } \\
\text { analysis }\end{array}$ & $80.48 \%$ & $94.58 \%$ \\
\hline $\begin{array}{l}\text { Result of discourse } \\
\text { structure analysis }\end{array}$ & $76.14 \%$ & $95.45 \%$ \\
\hline
\end{tabular}

Table 6. Results of the integrated analysis model

\section{Conclusion}

In this paper, we propose a statistical dialogue analysis model which can perform both speech act analysis and discourse structure analysis using maximum entropy model. The model can automatically acquire discourse knowledge from a discourse tagged corpus to resolve ambiguities. We defined the DSBs to represent the structural relationship of discourse between two consecutive utterances in a dialogue and used them for statistically analyzing both the speech act of an utterance and the discourse structure of a dialogue. By using the separated feature functions together with the unified feature functions, we could alleviate the data sparseness problems to improve the system performance. The model can, we believe, analyze dialogues more effectively than other previous works because it manages speech act analysis and discourse structure analysis at the same time using the same framework.

\section{Acknowledgements}

Authors are grateful to the anonymous reviewer for their valuable comments on this paper. Without their comments, we may miss important mistakes made in the original draft.

\section{References}

Andernach, T. 1996. A Machine Learning Approach to the Classification of Dialogue Utterances. Proceedings of NeMLaP-2.

Berger, Adam L., Stephen A. Della Pietra, and Vincent J. Della Pietra. 1996. A Maximum Entropy Approach to Natural Language Processing. Computational Linguistics, 22(1):39-71.

Caberry, Sandra. 1989. A Pragmatics-Based Approach to Ellipsis Resolution. Computational Linguistics, 15(2):75-96.

Carniak, Eugene. 1993. Statistical Language Learning. A Bradford Book, The MIT Press, Cambridge, Massachusetts, London, England.

Collins, M. J. 1996. A New Statistical Parser Based on Bigram Lexical Dependencies. Proceedings of the 34th Annual Meeting of the Association for Computational Linguistics, pages 184-191.

Grosz, Barbara J. and Candace L. Sidner. 1986. Attention, Intentions, and the Structure of Discourse. Computational Linguistics, 12(3):175204.

Hinkelman, E. A. 1990. Linguistic and Pragmatic Constraints on Utterance Interpretation. Ph.D. Dissertation, University of Rochester, Rochester, New York.

Hinkelman, E. A. and J. F. Allen. 1989. Two Constraints on Speech Act Ambiguity. Proceedings of the 27th Annual Meeting of the Association of Computational Linguistics, pages 212-219.

Kim, Chang-Hyun, Jae-Hoon Kim, Jungyun Seo, and Gil Chang Kim. 1994. A Right-to-Left Chart 
Parsing for Dependency Grammar using Headable Path. Proceeding of the 1994 International Conference on Computer Processing of Oriental Languages (ICCPOL), pages 175-180.

Lambert, Lynn. 1993. Recognizing Complex Discourse Acts: A Tripartite Plan-Based Model of Dialogue. Ph.D. Dissertation, The University of Delaware, Newark, Delaware.

Lambert, Lynn and Sandra Caberry. 1991. A Tripartite Plan-based Model of Dialogue. Proceedings of $A C L$, pages 47-54.

Lee, Jae-won, Jungyun Seo, Gil Chang Kim. 1997. A Dialogue Analysis Model With Statistical Speech Act Processing For Dialogue Machine Translation. Proceedings of Spoken Language Translation (Workshop in conjunction with (E)ACL'97), pages 10-15.

Lee, Hyunjung, Jae-Won Lee and Jungyun Seo. 1998. Speech Act Analysis Model of Korean Utterances for Automatic Dialog Translation. Journal of Korea Information Science Society (B): Software and Applications, 25(10):1443-1552 (In Korean).

Litman, Diane J. and James F. Allen. 1987. A Plan Recognition Model for Subdialogues in Conversations. Cognitive Science, pages 163-200.

Nagata, M. and T. Morimoto. 1994a. First steps toward statistical modeling of dialogue to predict the speech act type of the next utterance. Speech Communication, 15: 193-203.

Nagata, M. and T. Morimoto. 1994b. An information-theoretic model of discourse for next utterance type prediction. Transactions of Information Processing Society of Japan, 35(6):1050-1061.

Reithinger, N. and M. Klesen. 1997. Dialogue act classification using language models. Proceedings of EuroSpeech-97, pages 2235-2238.

Reynar, J. C. and A. Ratnaparkhi. 1997. A Maximum Entropy Approach to Identifying Sentence Boundaries. In Proceeding of the Fifth Conference on Applied Natural Language Processing, pages 16-19.

Ristad, E. 1996. Maximum Entropy Modeling Toolkit. Technical Report, Department of Computer Science, Princeton University.

Samuel, Ken, Sandra Caberry, and K. Vijay-Shanker. 1998. Computing Dialogue Acts from Features with Transformation-Based Learning. Applying Machine Learning to Discourse Processing: Papers from the 1998 AAAI Spring Symposium. Stanford, California. Pages 90-97.

Walker, Marilyn A. 1996. Limited Attention and Discourse Structure. Computational Linguistics, 22(2):255-264. 